\title{
Near Earth Asteroids with measurable Yarkovsky effect
}

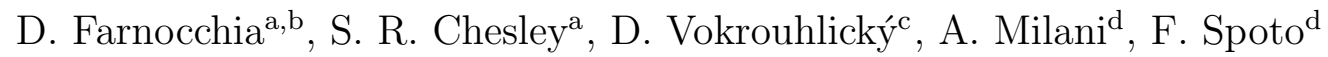 \\ ${ }^{a}$ Jet Propulsion Laboratory/Caltech, 4800 Oak Grove Drive, Pasadena, CA 91109, USA \\ ${ }^{b}$ SpaceDyS, Via Mario Giuntini 63, 56023 Cascina, Pisa, Italy \\ ${ }^{c}$ Institute of Astronomy, Charles University, V Holešovičkách 2, CZ-18000 Prague 8, \\ Czech Republic \\ ${ }^{d}$ Department of Mathematics, University of Pisa, Largo Pontecorvo 5, 56127 Pisa, Italy
}

\begin{abstract}
We seek evidence of the Yarkovsky effect among Near Earth Asteroids (NEAs) by measuring the Yarkovsky-related orbital drift from the orbital fit. To prevent the occurrence of unreliable detections we employ a high precision dynamical model, including the Newtonian attraction of 16 massive asteroids and the planetary relativistic terms, and a suitable astrometric data treatment. We find 21 NEAs whose orbital fits show a measurable orbital drift with a signal to noise ratio (SNR) greater than 3 . The best determination is for asteroid (101955) $1999 \mathrm{RQ}_{36}$, resulting in the recovery of one radar apparition and an orbit improvement by two orders of magnitude. In addition, we find 16 cases with a lower SNR that, despite being less reliable, are good candidates for becoming stronger detections in the future. In some cases it is possible to constrain physical quantities otherwise unknown by means of the detected orbital drift. Furthermore, the distribution of the detected orbital drifts shows an excess of retrograde rotators that can be connected to the delivery mechanism from the most important NEA feeding resonances and allows us to infer the distribution for NEAs obliquity. We discuss the implications of the Yarkovsky effect for impact predictions. In particular, for asteroid (29075) 1950 DA our results favor a retrograde rotation that would rule out an impact in 2880 .
\end{abstract}

Keywords: Asteroids, dynamics, Celestial mechanics, Near-Earth objects, Orbit determination

Email address: Davide.Farnocchia@jpl.nasa.gov (D. Farnocchia) 


\section{Introduction}

It is well known that nongravitational forces should be considered as important as collisions and gravitational perturbations for the overall understanding of asteroid evolution (Bottke et al., 2006). The most important nongravitational perturbation is the Yarkovsky effect, which is due to radiative recoil of anisotropic thermal emission and causes asteroids to undergo a secular semimajor axis drift $d a / d t$. Typical values of $d a / d t$ for sub-kilometer NEAs are $10^{-4}-10^{-3} \mathrm{au} / \mathrm{Myr}$ (Vokrouhlický et al., 2000).

The Yarkovsky acceleration depends on several physical quantities such as spin state, size, mass, shape, and thermal properties (Vokrouhlický, 1999). Furthermore, Rozitis and Green (2012) show that surface roughness also plays an important role by enhancing the Yarkovsky related semimajor axis drift by as much as tens of per cent. Though no complete physical characterization is typically available to compute the Yarkovsky acceleration based on a thermophysical model, the orbital drift may be detectable from an astrometric dataset. As a matter of fact, a purely gravitational dynamics could result in an unsatisfactory fit to the observational data. This is especially true when extremely accurate observations are available, e.g., radar observations, or when the observational dataset spans a long time interval thus allowing the orbital drift to accumulate and become detectable.

Until recently, the Yarkovsky effect has been measured directly only in three cases, (6489) Golevka (Chesley et al.,, 2003), (152563) 1992 BF (Vokrouhlický et al., 2008), and recently for (101955) $1999 \mathrm{RQ}_{36}$ (Chesley et al., 2012). For both Golevka and $1999 \mathrm{RQ}_{36}$ the Yarkovsky perturbation must be included to fit accurate radar observations spanning three apparitions. For 1992 BF the Yarkovsky effect is needed to link 4 precovery observations of 1953. Furthermore, in the case of $1999 \mathrm{RQ}_{36}$ the available physical characterization, along with the estimate of the Yarkovsky effect, allows the estimate of the asteroid's bulk density.

Nugent et al. (2012b) find 54 detections of semimajor axis drift by performing a search for semimajor axis drift among NEAs similar to the one presented in this paper. However, there are differences in the observational data treatment, in the modeling, and in the selection filters. A description of the differences and a comparison of the results is contained in Sec. 3.2. Nugent et al. (2012a) use WISE-derived geometric albedos and diameters to predict orbital drifts for 540 NEAs. Even if none of these objects has an observational record that allows one to measure the predicted orbital drift, the 
authors list upcoming observing opportunities that may reveal the Yarkovsky signal.

The Yarkovsky effect plays an important role for orbital predictions such as those concerning Earth impacts. In particular, when an asteroid has an exceptionally well constrained orbit, the Yarkovsky effect may become the principal source of uncertainty. Milani et al. (2009) show how the size of the semimajor axis drift along with its uncertainty modifies impact predictions for the next century for $1999 \mathrm{RQ}_{36}$. The cumulative impact probability is approximately $10^{-3}$, while a Yarkovsky-free propagation would rule out any impact event. Chesley et al. (2012) improve the $d a / d t$ estimate by means of September 2011 Arecibo radar measurements and find a cumulative impact probability approximately $4 \times 10^{-4}$. Another remarkable case is (99942) Apophis. Though only a marginal $d a / d t$ estimate is available, Giorgini et al. (2008) and Chesley et al. (2009) prove that the occurrence of an impact in 2036 is decisively driven by the magnitude of the Yarkovsky effect. On the longer term, Giorgini et al. (2002) show that an impact between asteroid (29075) 1950 DA and the Earth in 2880 depends on the accelerations arising from thermal re-radiation of solar energy absorbed by the asteroid.

\section{Methodology}

\subsection{Yarkovsky modeling and determination}

The Yarkovsky effect depends on typically unknown physical quantities. As the primary manifestation is a semimajor axis drift, we seek a formulation depending on a single parameter to be determined as a result of the orbital fit. To bypass the need of physical characterization we used a comet-like model (Marsden et al., 1973) for transverse acceleration $a_{t}=A_{2} g(r)$, where $g$ is a suitable function of the heliocentric distance $r$ and $A_{2}$ is an unknown parameter. To determine $A_{2}$ we used a 7 -dimensional differential corrector: starting from the observational dataset we simultaneously determine a bestfitting solution for both the orbital elements and $A_{2}$.

Once $A_{2}$ is determined from the orbital fit we estimate semimajor axis drift by means of Gauss' perturbative equations:

$$
\dot{a}=\frac{2 a \sqrt{1-e^{2}}}{n r} A_{2} g(r)
$$

where $a$ is the semimajor axis, $e$ is the eccentricity and $n$ is the mean motion. 
By averaging we obtain

$$
\overline{\dot{a}}=\frac{a \sqrt{1-e^{2}} A_{2}}{\pi} \int_{0}^{T} \frac{g(r)}{r} d t=\frac{A_{2}}{\pi n a} \int_{0}^{2 \pi} r g(r) d f
$$

where $T$ is the orbital period and $f$ is the true anomaly. Let us now assume $g(r)=\left(r_{0} / r\right)^{d}$, where $r_{0}$ is a normalizing parameter, e.g., we use $r_{0}=1$ au. In this case the semimajor axis drift is

$$
\overline{\dot{a}}=\frac{A_{2}\left(1-e^{2}\right)}{\pi n}\left(\frac{r_{0}}{p}\right)^{d} \int_{0}^{2 \pi}(1+e \cos f)^{d-1} d f .
$$

By Taylor expansion, we have

$$
\int_{0}^{2 \pi}(1+e \cos f)^{d-1} d f=\sum_{k=0}^{\infty}\left(\begin{array}{c}
d-1 \\
k
\end{array}\right) e^{k} \int_{0}^{2 \pi} \cos ^{k} f d f .
$$

The odd powers of the cosine average out, so we obtain

$$
\overline{\dot{a}}=\frac{2 A_{2}\left(1-e^{2}\right)}{n}\left(\frac{r_{0}}{p}\right)^{d} J(e, d)
$$

where

$$
J(e, d)=\sum_{k=0}^{\infty} \alpha_{k} e^{2 k} \quad, \quad \alpha_{k}=\left(\begin{array}{c}
d-1 \\
2 k
\end{array}\right)\left(\begin{array}{c}
2 k \\
k
\end{array}\right) \frac{1}{2^{2 k}} .
$$

The ratio

$$
\frac{\alpha_{k+1}}{\alpha_{k}}=\left(1-\frac{d+1}{2 k+2}\right)\left(1-\frac{d}{2 k+2}\right)
$$

is smaller than 1 for $d>0$ and $k$ large enough. Therefore, $\alpha_{k}$ are bounded and $J(e, d)$ is convergent for any $e<1$. Equation (7) can be used to recursively compute $\alpha_{k}$ starting from $\alpha_{0}=1$. For integer $d$ the series $J$ is a finite sum that can be computed analytically, e.g., $J(e, 2)=1$ and $J(e, 3)=1+0.5 e^{2}$.

The proper value of $d$ is not easily determined. From Vokrouhlický (1998), we have

$$
a_{t} \simeq \frac{4(1-A)}{9} \Phi(r) f(\Theta) \cos \gamma \quad, \quad f(\Theta)=\frac{0.5 \Theta}{1+\Theta+0.5 \Theta^{2}}
$$

for the Yarkovsky diurnal component (which is typically dominant), where $A$ is the Bond albedo, $\Theta$ is the thermal parameter, $\gamma$ is the obliquity, and 
$\Phi(r)$ is the standard radiation force factor, which is inversely proportional to the bulk density $\rho$, the diameter $D$, and $r^{2}$. The thermal parameter $\Theta$ is related to the thermal inertia $\Gamma$ by means of the following equation

$$
\Theta=\frac{\Gamma}{\varepsilon \sigma T_{*}^{3}} \sqrt{\frac{2 \pi}{P}}
$$

where $\varepsilon$ is the emissivity, $\sigma$ is the Boltzmann's constant, $T_{*}$ is the subsolar temperature, and $P$ is the rotation period. In this paper we use $d=2$ to match the level of absorbed solar radiation. Then, from Eq. (8) we have that

$$
A_{2} \simeq \frac{4(1-A)}{9} \Phi(1 \mathrm{au}) f(\Theta) \cos \gamma .
$$

However, as $T_{*} \propto r^{-0.5}$ we have that $\Theta \propto r^{1.5}$, therefore the best value of $d$ depends on the object's thermal properties:

- for $\Theta \gg 1$ we obtain $f \propto r^{-1.5}$, which gives $d=3.5$;

- for $\Theta \ll 1$ we obtain $f \propto r^{1.5}$, which gives $d=0.5$.

These are limit cases, the true $d$ is always going to be between them. As a matter of fact, it turns out that most NEAs, whose rotation period is not excessively large and whose surface thermal inertia is not excessively small or large, have typically values of $\Theta$ near unity or only slightly larger, and we can thus expect $d$ value in the range $2-3$. As an example, Chesley et al. (2012) show that for $1999 \mathrm{RQ}_{36}$ the best match to the Yarkovsky perturbation computed by using a linear heat diffusion model is $d=2.75$.

What matters to us is that $d a / d t$ does not critically depend on the chosen value of $d$. As an example for asteroid $1999 \mathrm{RQ}_{36}$ we have that $d a / d t=$ $(-18.99 \pm 0.10) \times 10^{-4} \mathrm{au} / \mathrm{Myr}$ for $d=2$ and $d a / d t=(-19.02 \pm 0.10) \times 10^{-4}$ $\mathrm{au} / \mathrm{Myr}$ for $d=3$. Another example is Golevka, for which we obtain $d a / d t=$ $(-6.62 \pm 0.64) \times 10^{-4} \mathrm{au} / \mathrm{Myr}$ for $d=2$ and $d a / d t=(-6.87 \pm 0.66) \times 10^{-4}$ $\mathrm{au} / \mathrm{Myr}$ for $d=3$. In both cases the difference in $d a / d t$ due to the different values assumed for $d$ is well within one standard deviation.

\subsection{Dynamical model}

To consistently detect the Yarkovsky effect we need to account for the other accelerations down to the same order of magnitude. For a sub-kilometer NEA, typical values of $a_{t}$ range from $10^{-15}$ to $10^{-13} \mathrm{au} / \mathrm{d}^{2}$. 
Table 1: Gravitational parameters of perturbing asteroids. The masses of Ceres, Pallas, and Vesta are from Standish and Hellings (1989), the ones of Euphrosyne and Herculina are from the Institute of Applied Astronomy of RAS, St. Petersburg, Russia (http://www.ipa.nw.ru/PAGE/DEPFUND/LSBSS/engmasses.htm), the mass of Juno is from Konopliv et al. (2011), all the others are from Baer et al. (2011).

\begin{tabular}{lc}
\hline \hline Asteroid & $\mathrm{Gm}\left[\mathrm{km}^{3} / \mathrm{s}^{2}\right]$ \\
\hline (1) Ceres & 63.200 \\
(2) Pallas & 14.300 \\
(4) Vesta & 17.800 \\
(10) Hygea & 6.0250 \\
(29) Amphitrite & 1.3271 \\
(511) Davida & 3.9548 \\
(65) Cybele & 1.0086 \\
(9) Metis & 1.3669 \\
(15) Eunomia & 2.2295 \\
(31) Euphrosyne & 1.1280 \\
(52) Europa & 1.2952 \\
(704) Interamnia & 4.7510 \\
(16) Psyche & 1.7120 \\
(3) Juno & 1.9774 \\
(532) Herculina & 1.5262 \\
(87) Sylvia & 1.3138 \\
\hline
\end{tabular}

Our N-body model includes the Newtonian accelerations of the Sun, eight planets, the Moon, and Pluto that are based on JPL's DE405 planetary ephemerides (Standish, 2000). Furthermore, we added the contribution of 16 massive asteroids, as listed in Table 1 .

We used a full relativistic force model including the contribution of the Sun, the planets, and the Moon. Namely, we used the Einstein-InfeldHoffman (EIH) approximation as described in Moyer (2003) or Will (1993). As already noted in Chesley et al. (2012), the relativistic term of the Earth should not be neglected because of significant short range effects during Earth approaches that NEAs may experience. For asteroids with a large perihelion distance such as Golevka also Jupiter's term could be relevant. Figure 1 compares the relativistic accelerations of the Earth and Jupiter as they formally appear in the EIH equations of motion to the transverse Yarkovsky acceleration acting on Golevka. 


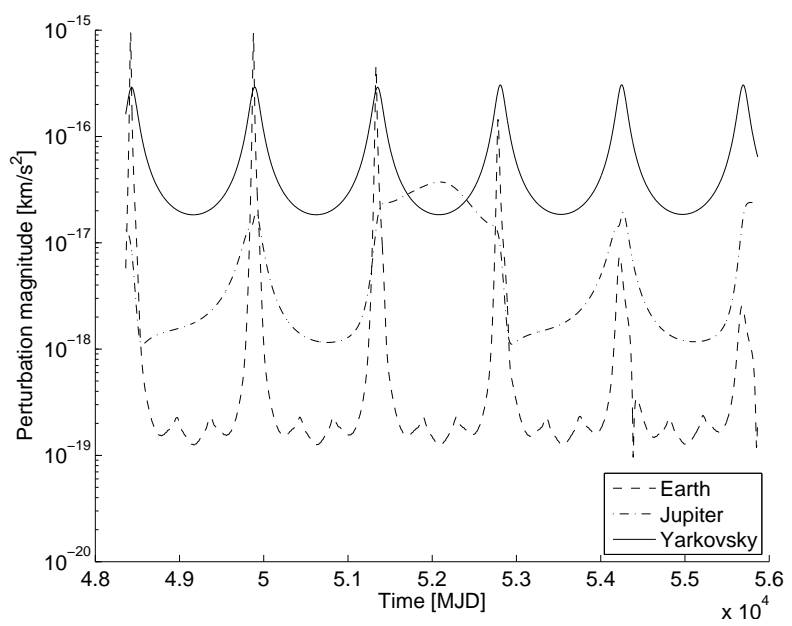

Figure 1: Relativistic accelerations of the Earth and Jupiter as they formally appear in the EIH equations of motion compared to the transverse Yarkovsky acceleration acting on Golevka.

\subsection{Observational error model}

The successful detection of the Yarkovsky effect as a result of the orbital fit strongly depends on the quality of the observations involved. In particular, the availability of radar data is often decisive due to the superior relative accuracy of radar data with respect to optical ones. Moreover, radar measurements are orthogonal to optical observations: range and range rate vs. angular position in the sky.

Since the Yarkovsky effect acts as a secular drift on semimajor axis we have a quadratic effect in mean anomaly: the longer the time span the stronger the signal. However, the presence of biases in historical data and unrealistic weighting of observations may lead to inaccurate results. To deal with this problem we applied the debiasing and weighting scheme described in Chesley et al. (2010). This scheme is a valid error model for CCD observations, while for pre-CCD data the lack of star catalog information and the very uneven quality of the observations represents a critical problem. In these cases the occurrence of unrealistic nominal values for Yarkovsky model parameters presumably point to bad astrometric treatments and have to be rejected.

To prevent outliers from spoiling orbital fits, we applied the outlier rejection procedure as described in Carpino et al. (2003).

Besides the astrometric treatment described above, in the following cases 
we applied an ad hoc observation weighting:

- $1999 \mathrm{RQ}_{36}$ : as already explained by Chesley et al. (2012), in some cases there are batches containing an excess of observations from a single observatory in a single night. To reduce the effect of these batches to a preferred contribution of 5 observations per night, we relaxed the weight by a factor $\sqrt{N / 5}$, where $N$ is the number of observations contained in the batch.

- $1992 \mathrm{BF}$ : as the precovery observations of 1953 have been carefully remeasured in Vokrouhlický et al. (2008), these observations were given a weight 0.5 " in right ascension and 1 " in declination.

- Apollo: the large dataset available for Apollo contains observations going back to 1930. Many pre-CCD era observation batches show unusually high residuals, especially during close Earth approches. To lower the effect of non-CCD observation, we used weights 10" for observations from 1930 to 1950 and 3" from 1950 to 1990.

- 1989 ML: the discovery apparition contains observations from Palomar Mountain showing large residuals whether or not the Yarkovsky perturbation is included in the model. Even if this apparition increases the observed arc by three years only, we felt it safer to weight the corresponding observations 3".

\subsection{Treatment of precovery observations}

There are a few cases where the Yarkovsky signal is mainly contained in few isolated precovery observations. This is the case of the already mentioned asteroid $1992 \mathrm{BF}$, which has 4 isolated observations in 1953 from Palomar Mountain DSS. Other cases are

- $1999 \mathrm{FK}_{21}$, which has 6 isolated observations in 1971 from Palomar Mountain;

- $2001 \mathrm{MQ}_{3}$, which has 4 isolated observations in 1951 from Palomar Mountain DSS;

- 1989 UQ, which has 3 isolated observations in 1954 from Palomar Mountain; 

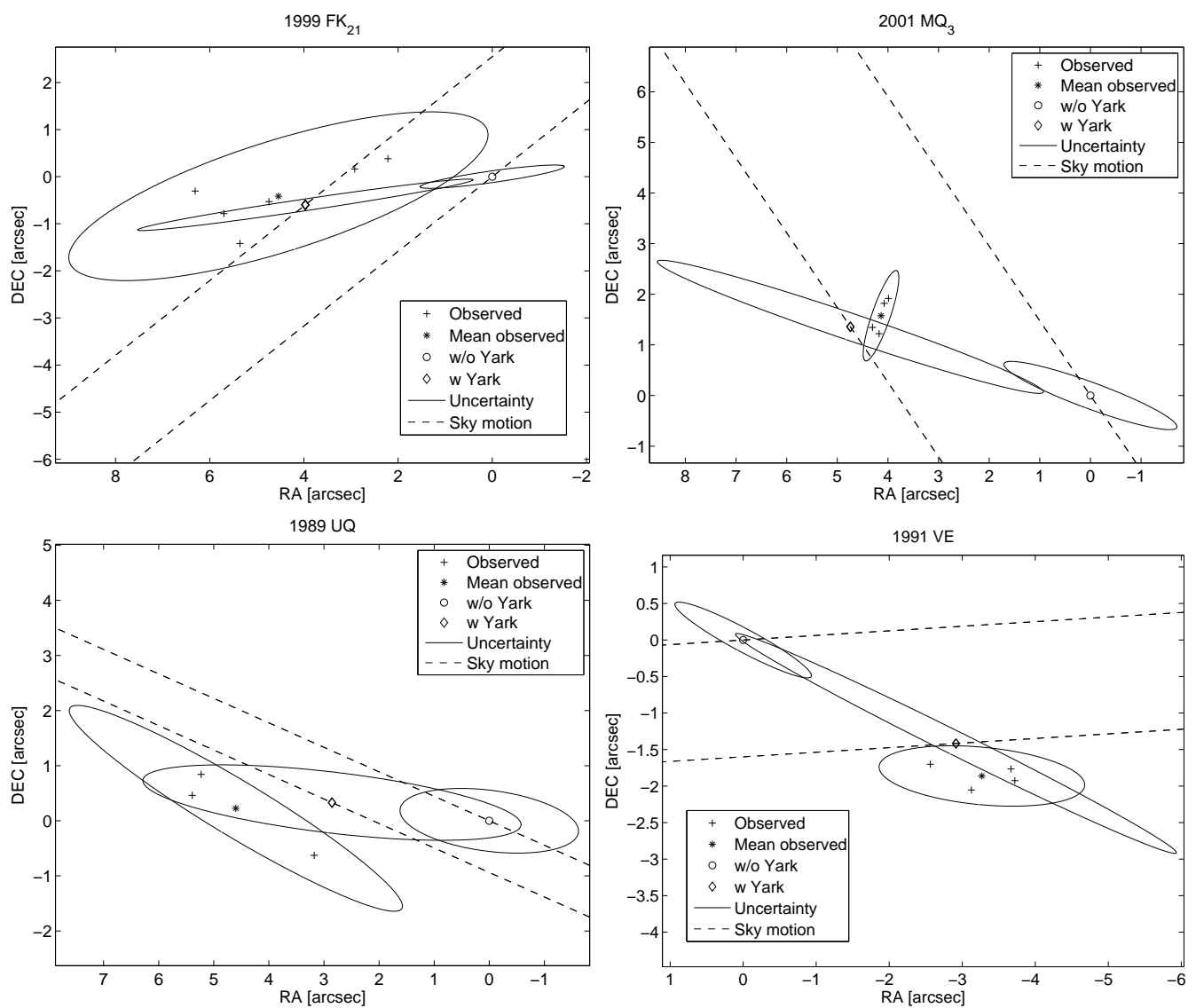

Figure 2: Observed position, average observed position, and predicted postfit position with the Yarkovsky perturbation in the dynamics with respect to the predictions without the Yarkovsky perturbation in the dynamics. The uncertainty ellipses for the mean observed position and the predicted positions correspond to the $3-\sigma$ level. The dashed lines represent the predicted motion in the sky of the asteroid in the precovery apparition. Circles are the predicted positions, crosses are the measured positions, stars are the mean of the measured positions, while diamonds are the predicted positions when the Yarkovsky perturbation is included in the dynamics. The origin is arbitrarily set to the non-Yarkovsky prediction.

- $1991 \mathrm{VE}$, which has 4 isolated observations in 1954 from Palomar Mountain DSS.

For all these cases it would be desirable to remeasure the precovery observations as done for $1992 \mathrm{BF}$ in Vokrouhlický et al. (2008), where precovery observations were corrected by an amount up to 3.1". For this reason we conservatively gave weights 3 " to the precovery observations of the four asteroids 
above.

Besides the conservative weighting, we ruled out clock error as a possible cause of the Yarkovsky signal. Figure 2 shows the scenario for the four mentioned asteroids during the precovery apparition. We can see that it is not possible to match the observations by translating the non-Yarkovsky uncertainty ellipse on the along track direction. The Yarkovsky solution produces a shift in the weak direction that give a better match to the observations, in particular when we take the average of the observed positions.

\subsection{Filtering spurious results}

To assess the reliability of the Yarkovsky detections we computed an expected value for $A_{2}$ starting from the 1999 RQ ${ }_{36}$ case, which is the strongest and most reliable detection, by scaling according to (10)

$$
\left(A_{2}\right)_{\text {exp }}=\left(A_{2}\right)_{R Q 36} \frac{D_{R Q 36}}{D} .
$$

For diameter $D$ we used either the known value when available or an assumed value computed from the absolute magnitude $H$ according to the following relationship (Pravec and Harris, 2007):

$$
D=1329 \mathrm{~km} \times \frac{10^{-0.2 H}}{\sqrt{p_{V}}}
$$

where $p_{V}$ is the albedo, assumed to be 0.154 if unknown, in agreement with the Palermo scale computation (Chesley et al., 2002).

Some physical properties of $1999 \mathrm{RQ}_{36}$ maximize $A_{2}$ (Chesley et al., 2012). In particular $\gamma$ is nearly $180^{\circ}, A$ is 0.01 , and $\rho$ is low $\left(0.96 \mathrm{~g} / \mathrm{cm}^{3}\right)$. On the other hand $\Theta=4.33$ for which $f(\Theta) \simeq 0.15$ while the maximum is $\simeq 0.21$. For these reasons we selected those Yarkovsky detections for which $\mathcal{S}=\left|A_{2} /\left(A_{2}\right)_{\text {exp }}\right|$ was smaller than 1.5. The selected threshold allows some tolerance as we are scaling only by $D$ without accounting for other quantities such as bulk density, thermal properties, obliquity, spin rate, and surface roughness.

A high SNR threshold is likely to produce robust detections with respect to the astrometric data treatment. With lower SNR the sensitivity to the observation error model increases and detections become less reliable. We decided that 3 was a sensible choice for minimum SNR, even if we analyze detections with smaller SNR in Sec. 3.3. 


\section{Results}

We applied our 7-dimensional differential corrector to determine the parameter $A_{2}$ and the corresponding $d a / d t$ for all known NEAs. After applying the filters discussed in Sec. 2.5 we obtain 21 Yarkovsky detections that we consider reliable (Table 2). The reported uncertainties are marginal, i.e., they fully take into account the correlation between $A_{2}$ (and thus $d a / d t$ ) and the orbital elements.

We cross-checked these detections by using two independent software suites: the JPL Comet and Asteroid Orbit Determination Package and OrbFit (http://adams.dm.unipi.it/orbfit/)1: in all cases we found agreement at better than the $1-\sigma$ level.

\subsection{BD}

Asteroid 2009 BD is very small and to fit its observational dataset it is necessary to include solar radiation pressure (Micheli et al., 2012). Therefore, we also included in the model a radial acceleration $a_{r}=A_{1} / r^{2}$. Along with the tabulated value of $A_{2}$, we obtained $A_{1}=(62.05 \pm 8.85) \times 10^{-12} \mathrm{au} / \mathrm{d}^{2}$. This results in an area to mass ratio $A / M=(2.72 \pm 0.39) \times 10^{-4} \mathrm{~m}^{3} / \mathrm{kg}$, which is consistent at the $1-\sigma$ level with the value reported by Micheli et al. (2012), i.e., $(2.97 \pm 0.33) \times 10^{-4} \mathrm{~m}^{3} / \mathrm{kg}$.

\subsection{Comparison with other published results}

The first three objects of Table 2 are the already known cases of Golevka, $1992 \mathrm{BF}$, and $1999 \mathrm{RQ}_{36}$. While for $1999 \mathrm{RQ}_{36}$ there is a perfect match between our result and the one in Cheslev et al. (2012), for Golevka and 1992 BF the values are different from Chesley et al. (2003) and Vokrouhlický et al. (2008), respectively. However, this can be easily explained by the availability of new astronomy and the fact that the present paper adopted the debiasing and weighting scheme by Chesley et al. (2010), which was not available at the time of the earlier publications.

As already mentioned, Nugent et al. (2012b) performed a search similar to the one presented in this paper and found 54 NEAs with a measurable semimajor axis drift. The main differences are the following:

\footnotetext{
${ }^{1}$ OrbFit was used in the development version 4.3 , currently in beta-testing.
} 
- They selected only numbered objects, while we included all known NEAs.

- Their observation dataset was slightly different as they used observations until 2012 January 31, while we have data until 2012 October 31. This difference does not really matter for optical data, but it does for radar data, e.g., for Aten and Toro. Moreover, they did not use single apparition radar, while we did as we think they represent an important constraint.

- They solved for constant $d a / d t$ while we used constant $A_{2}$ and then convert to $d a / d t$. These techniques are equivalent when the semimajor axis and eccentricity are constant, but there could be differences as we cannot assume $d a / d t$ constant for objects experiencing deep planetary close approaches.

- They searched for the best-fit $d a / d t$ by means of the golden section algorithm, i.e., they computed the RMS of the residuals corresponding to the best fitting orbital elements for fixed values of $d a / d t$, then the minimum is obtained by interpolation. In this paper we used a full 7-dimensional differential corrector. The two methods should be equivalent.

- They used 1 as lower bound for SNR, while we use 3 that gives detections more robust against changes in the observation weighting. Also, they used the "sensitivity" parameter, i.e., a metric to measure the sensitivity of a dataset to the presence of a semimajor axis drift. We did not use such a metric as we think that an $\mathrm{SNR} \geq 3$ is already a good metric in that respect.

- We kept only those objects for which the measured orbital drift can be related to the Yarkovsky perturbation presuming that inconsistencies stem from astrometric errors, while they also considered the possibility of other nongravitational effects such as a loss of mass.

Table 2 contains a comparison between our orbital drifts and the ones reported by Nugent et al. (2012b). 2009 BD, 1999 MN, 1999 FA, and 2005 $\mathrm{ES}_{70}$ are present only in our list as they are not numbered, while $2001 \mathrm{MQ}_{3}$, 1989 UQ, and $1991 \mathrm{VE}$ are eliminated by their filters. It is worth pointing out that also $1999 \mathrm{RQ}_{36}, 1992 \mathrm{BF}$, Golevka, and YORP have been filtered out 
by Nugent et al. (2012b) criteria, even though they report the corresponding detections for a comparison with Cheslev et al. (2008).

Among the cases that Nugent et al. (2012b) report with SNR $>3$ we neglected the following three:

- (1036) Ganymed for which we found $A_{2}=(-16.54 \pm 4.35) \times 10^{-15} \mathrm{au} / \mathrm{d}^{2}$, corresponding to $d a / d t=(-6.06 \pm 1.59) \times 10^{-4} \mathrm{au} /$ Myr. However, the nominal $A_{2}$ is 28 times larger than $\left(A_{2}\right)_{\text {exp }}$, so we marked this detection as spurious. As Ganymed observations go back to 1924, this unreliable detection might be due to bad quality astrometry.

- (4197) 1982 TA for which we used the radar apparition of 1996, which reduced the SNR below 1. For this object we found $A_{2}=(5.61 \pm$ $14.26) \times 10^{-15} \mathrm{au} / \mathrm{d}^{2}$, corresponding to $d a / d t=(3.88 \pm 9.86) \times 10^{-4}$ $\mathrm{au} /$ Myr. For comparison Nugent et al. (2012b) report $d a / d t=(30.9 \pm$ $9.2) \times 10^{-4} \mathrm{au} / \mathrm{Myr}$.

- (154330) $2002 \mathrm{VX}_{4}$ for which we found $\left.A_{2}=102.36 \pm 36.34\right) \times 10^{-15}$ $\mathrm{au} / \mathrm{d}^{2}$, corresponding to $d a / d t=(43.10 \pm 15.25) \times 10^{-4} \mathrm{au} /$ Myr. Again, the value of $A_{2}$ was $\sim 4$ times larger than $\left(A_{2}\right)_{\text {exp }}$, so also this detection was marked as spurious.

Besides the differences outlined above, there is an overall agreement for the common cases. As a matter of fact by computing the $\sigma$ of the difference, i.e., $\sigma^{2}=\sigma_{1}^{2}+\sigma_{2}^{2}$, there are only two cases that are not consistent at the 1- $\sigma$ level:

- 1992 BF, for which we used remeasured observations (Vokrouhlický et al., 2008) and changed the weights (see Sec. 2.3) for the 1953 apparition. The remeasurements are not available in the MPC database.

- Apollo, for which we applied a suitable manual weighting as described in Sec. 2.3.

\subsection{Lower SNR and other less reliable detections}

Table 3 contains detections that we rate as nonspurious on the basis of the $S$ ratio between expected and measured value, down to $\mathrm{SNR}=2$ plus the following remarkable cases: 


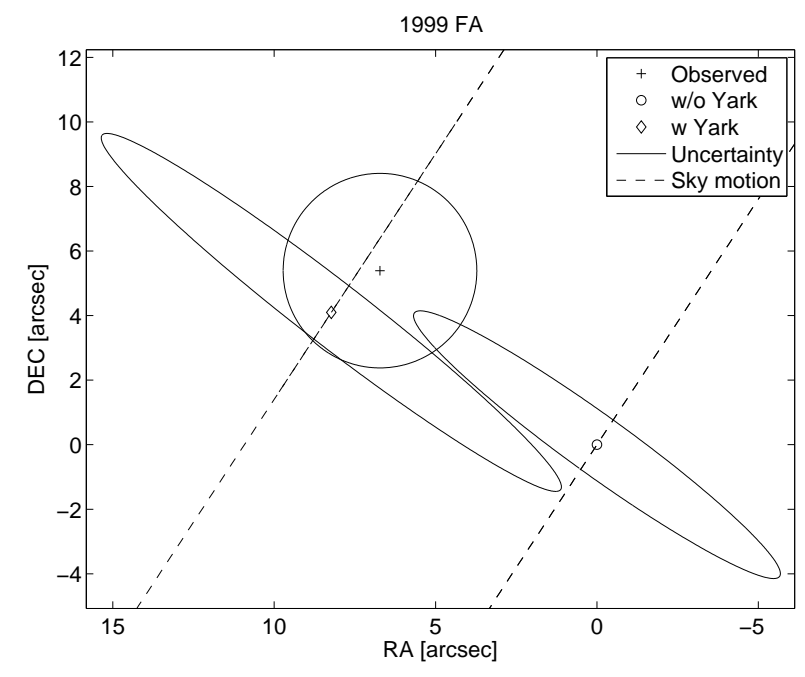

Figure 3: Same of Fig. 2 for asteroid 1999 FA. The uncertainty ellipses for predicted positions correspond to $3-\sigma$ levels, while the uncertainty for the observaed position correspond to $3 "$.

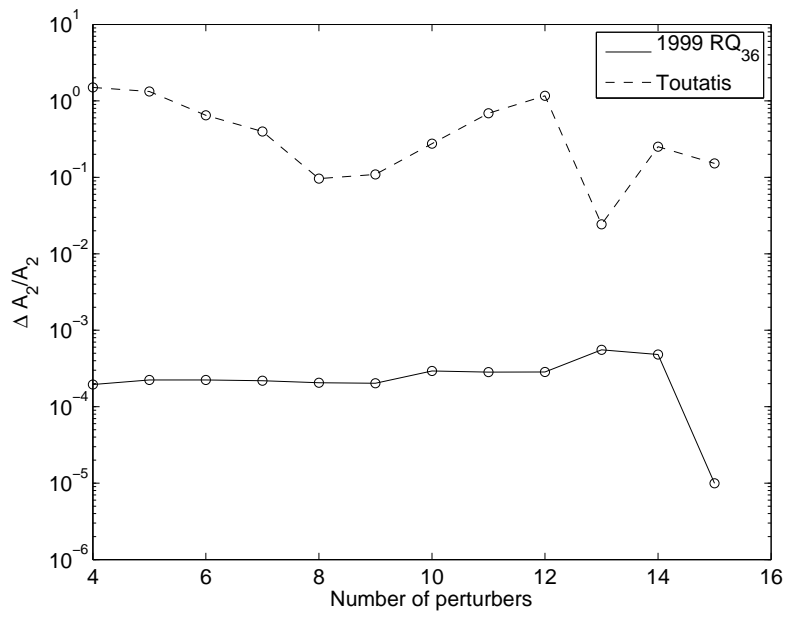

Figure 4: Relative displacement of $A_{2}$ with respect to the nominal value as a function of the number of perturbers for $1999 \mathrm{RQ}_{36}$ and Toutatis.

- 1999 FA, which has 1 isolated observation in 1978 from Siding Spring Observatory. By performing the same analysis of Sec. 2.4 (see Fig. 33) we see no clear improvement due to Yarkovsky as the observation is 3.5-4 $\sigma$ away from the prediction either way. In this case a clock error 
may bring the Yarkovsky solution close to the observation. As this detection depends on a lone, isolated observation we would rather be cautious and consider this detection reliable only if the 1978 observation is remeasured.

- Eros, which looks like a reliable detection as $\mathcal{S}=0.75$. However, the obliquity is known to be $89^{\circ}$ (Yeomans et al., 2000) and then we enter the regime where the seasonal component of the Yarkovsky effect is dominant. As the seasonal component is typically 10 times smaller then the diurnal one (Vokrouhlický et al., 2000) we mark this detection as spurious. This points to possible bad astrometric treatment, especially for historical observations dating back to 1893.

- Toutatis, which enters the Main Belt region because of the $4.12 \mathrm{au}$ aphelion and the low inclination $\left(0.44^{\circ}\right)$. Therefore, it is important to account for the uncertainty in the masses of the perturbing asteroids. By taking into account the uncertainty of the perturbing asteroid masses the actual uncertainty in $A_{2}$ increases by $11 \%$ with a commensurable drop in SNR. Figure 4 shows the evolution of $A_{2}$ as a function of the number of perturbers for asteroids $1999 \mathrm{RQ}_{36}$ and Toutatis. While for $1999 \mathrm{RQ}_{36}$ (aphelion $1.36 \mathrm{au}$ ) we reach convergence with just four perturbers, for Toutatis we have a quite irregular behavior suggesting that we may need to include more perturbers.

- $1994 \mathrm{XL}_{1}$, whose observations in 1994 from Siding Spring Observatory show high residual so we relaxed weights to 3".

- $2005 \mathrm{QC}_{5}$ and $2000 \mathrm{NL}_{10}$ have been included despite the low SNR. Similarly to the cases described in Sec. 2.4, we applied weights 3" to precovery observations and this data treatment weakened the Yarkovsky detection. However, we think that remeasuring the precovery observations may lead to reliable Yarkovsky detections for these objects.

- 1950 DA, for which the Yarkovsky effect plays an important role for impact predictions, e.g., see Sec. 4.4,

- Icarus, for which the low values of both the nominal value and the uncertainty put a strong contraint on $A_{2}$. In particular, it is worth pointing out that the constraint on $d a / d t$ is consistent with Vokrouhlický et al. (2000, Fig. 5) where $|d a / d t|<3 \times 10^{-4}$ au/Myr. 
There are other objects with an even lower SNR for which the Yarkovsky signal might be revealed if precovery observations were remeasured: (11284) Belenus, (66400) $1999 \mathrm{LT}_{7}$, (4688) $1980 \mathrm{WF}$, (67399) $2000 \mathrm{PJ}_{6}$, (267759) $2003 \mathrm{MC}_{7}$, and (88710) $2001 \mathrm{SL}_{9}$.

Though these detections have to be considered less reliable, some of them may be good candidates for becoming stronger detections in the future if high quality astrometry is obtained, e.g., by radar or GAIA (Mignard, 2003).

\section{Discussion}

\subsection{Connection with NEA feeding mechanisms}

The diurnal Yarkovsky effect produces a semimajor axis drift proportional to $\cos \gamma$ (Vokrouhlický et al., 2000). As the diurnal term is typically the dominant one, the sign of $d a / d t$ can be related to the asteroid spin orientation, i.e., a negative $d a / d t$ corresponds to a retrograde rotator while a positive $d a / d t$ corresponds to a prograde rotator. This conclusion is supported by the eight known obliquities for the asteroids in the sample that are listed in Table 2: in all cases the spin axis obliquity is consistent with the sign of $d a / d t$.

We can now use this interpretation and our solution for the Yarkovsky semimajor axis drift values for NEAs in the following way. Table 2 contains four prograde rotators and seventeen retrograde rotators. This excess of retrograde rotators can be explained by the nature of resonance feeding into the inner Solar System (Bottke et al., 2002). Most of the primary NEA source regions (e.g., the 3:1 resonance, JFCs, Outer Belt, etc.) allow main belt asteroids to enter by drifting either inwards or outwards, but the $\nu_{6}$ resonance is at the inner edge of the main belt and so asteroids can generally enter only by inwards drift, i.e., with retrograde rotation. Bottke et al. (2002) report that $37 \%$ of NEAs arrive via $\nu_{6}$ resonance. La Spina et al. (2004) point out that this implies $37 \%$ of NEAs have retrograde spin (via $\nu_{6}$ ), plus half of the complement (via other pathways). Thus, the retrograde fraction should be $0.37+0.5 \times 0.63=0.69$, while La Spina et al. $(2004)$ report $67 \%$ retrograde for their sample, which is dominated by large NEAs.

Table 2 contains $81 \%$ retrograde rotators, which is larger than $69 \%$ and then appears to be inconsistent with the theory. However, the asteroid sample of Table 2 is based on measured Yarkovsky mobility, and so is dominated 

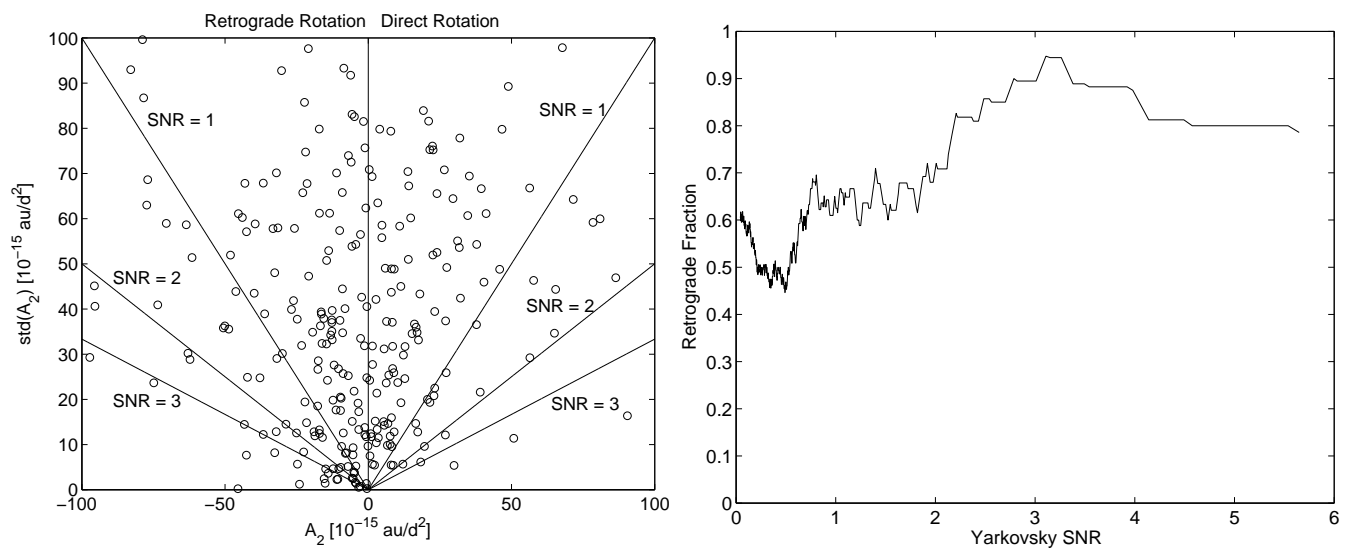

Figure 5: Left panel: distribution of $A_{2}$ and its uncertainty. Right side of plot is prograde rotation and left side is retrograde rotation. Right panel: running box mean in SNR for the fraction of asteroids with indication of retrograde spin. Cases with SNR $>2$ show a consistent 4:1 retrograde ratio, cases with little or no signal are split 50-50.

by small PHAs. For this population, 55\% are expected to arrive from $\nu_{6}$ according to the Bottke et al. (2002) NEO model (Bottke, personal communication), a much greater fraction than for NEAs as a whole. The same arithmetic used for NEAs gives $0.55+0.5 \times 0.45=0.78$, which matches very well the $81 \% \pm 8 \%$ value of Table 2 .

To assess the behavior of the fraction of retrograde rotators as a function of the SNR, we took all of the objects with $\mathcal{S}<1.5$. The left panel of Fig. 5 shows the distribution of $A_{2}$ and its uncertainty. The excess of retrograde rotators is clearly visible for $\mathrm{SNR}>3$ and also between 2 and 3, where small PHAs dominate. For lower SNR we have a more uniform distribution. The right panel of Fig. 5 is a running mean of the fraction of retrograde rotators as a function of the SNR. For SNR $<1$ we are in a noise dominated regime for which we have a rough $50 \%$ fraction of retrograde rotators, for $1<\mathrm{SNR}<2$ we have a transition from noise-dominated to signal dominated, and for SNR $>2$ we have a signal dominated regime with around $80 \%$ retrograde rotators.

We can also try to use the detected values to infer the obliquity distribution. From Eq. (10) we have that

$$
A_{2} \propto \frac{\cos \gamma}{D}
$$

and so we can estimate $\gamma$ by using the either known or estimated by Eq. (12) 


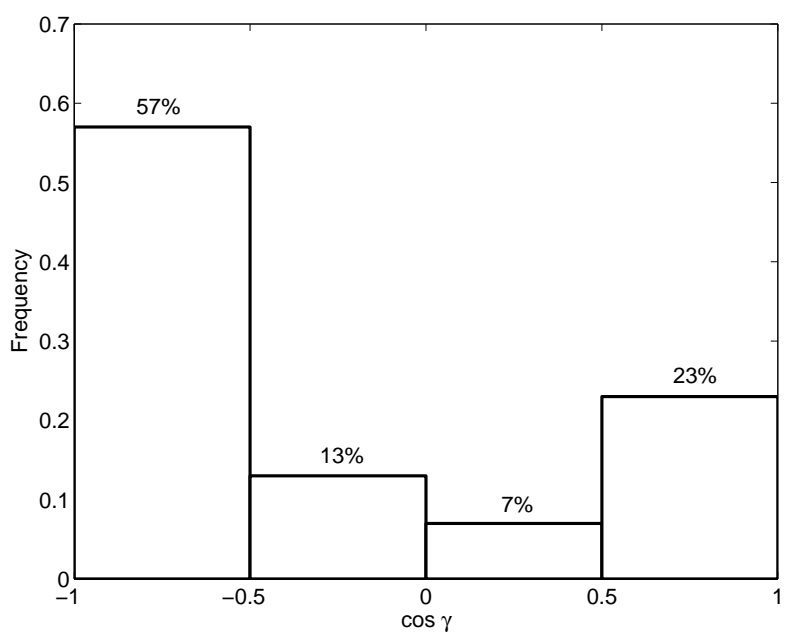

Figure 6: Inferred obliquity distribution for objects with SNR $>1$.

diameter and using $1999 \mathrm{RQ}_{36}$. By taking all the detections with $\mathrm{SNR}>1$ and assuming a fixed $\rho=1500 \mathrm{~kg} / \mathrm{m}^{3}$ we obtain the distribution of Fig. 6. where the cases with $|\cos \gamma|>1$ have been placed in the extreme obliquity bins. Despite the low number of bins, we can see the excess of retrograde rotators and the abundance of objects with an extreme obliquity, as expected from the YORP effect (Čapek and Vokrouhlický, 2004). While this distribution should be considered only approximate due to the numerous assumptions (e.g., neglecting dependence on bulk density, shape and thermal properties) we consider it to be a significant improvement over what is otherwise known. However, we find it interesting that it appears to be consistent with the observed obliquity distribution of the NEAs (La Spina et al., 2004).

\subsection{Spurious detections}

Our search for Yarkovsky signal produced a large number of spurious detections, i.e., semimajor axis drifts far larger than the Yarkovsky effect would cause. Figure 7 contains the histograms of $\mathcal{S}$ for different SNR intervals. For SNR $>3$ we have $67 \%$ spurious detections, for $2<$ SNR $<3$ we have $88 \%$.

It it worth trying to understand the reason of these spurious solutions. We think there are two possible causes:

- Bad astrometry treatment: as discussed in Sec. 2.3 and Sec. 2.4, nonCCD observations may contain errors that are difficult to model. If an ad hoc weighting is not used we may have misleading results. Indeed, 

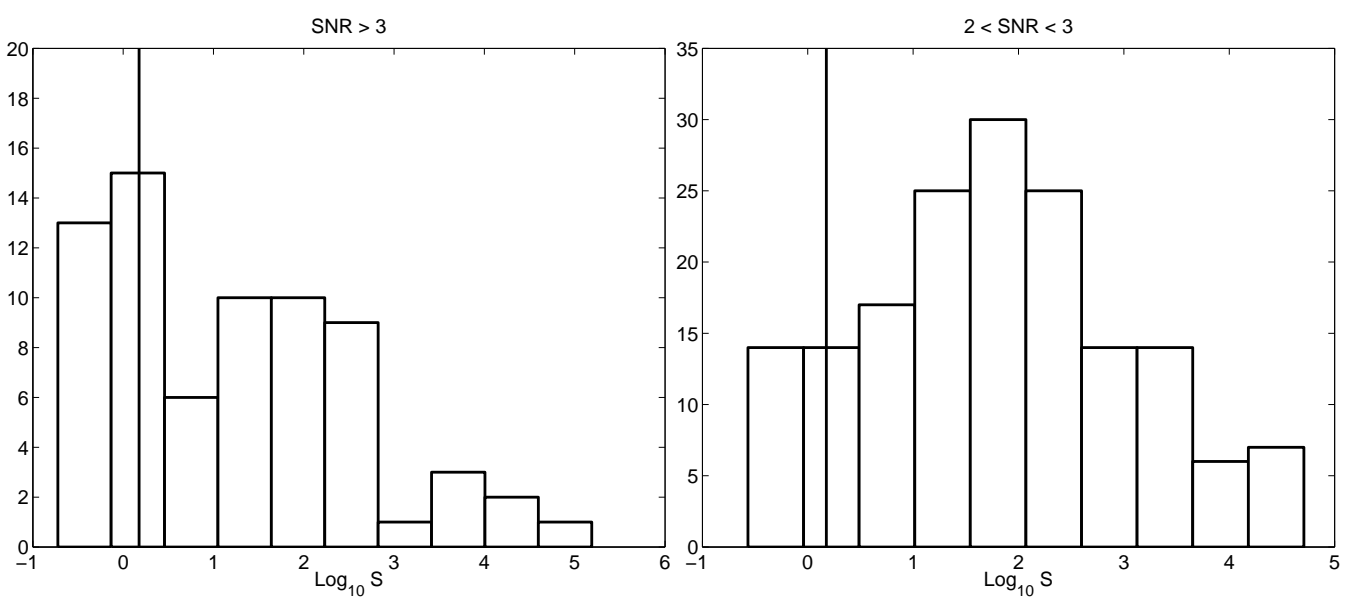

Figure 7: Histogram of $\mathcal{S}$ for different intervals of SNR. The vertical lines correspond to the selected threshold 1.5.

spot checking of such cases generally confirmed isolated astrometry as source of spurious detections.

- Incompleteness or inconsistency in the dynamical model: the formulation proposed in Sec. 2.1] is a simplified model of the Yarkovsky perturbation that might be poor in some cases. A more sophisticated formulation would require a rather complete physical characterization that is typically unavailable and thus cannot be used for a comprehensive search as done in this paper. Moreover, as discussed in Sec. 3.3. for Toutatis, we may need to include more perturbing asteroids (and the uncertainty in their masses) in the model. Finally, we cannot rule out the possibility of nongravitational perturbations different from Yarkovsky.

\subsection{Constraining physical quantities}

The results reported in Table 2 can be used to constrain physical quantities. When $A, D$, and $\gamma$ are known Eq. (10) provides a simple relationship between $\rho$ and $\Theta$. This relationship can be easily translated into a relationship between $\rho$ and the thermal inertia $\Gamma$ by means of Eq. (9). As a benchmark of these technique we can use asteroid 1999 RQ 36 (Fig. 8), for which the known values of $\Gamma$ and $\rho$ (Chesley et al., 2012) match the plotted contraint. Figure 9 shows the possible values of $\rho$ as a function of $\Gamma$ for asteroids Golevka, Apollo, Ra-Shalom, Toro, YORP, and Geographos. For the 


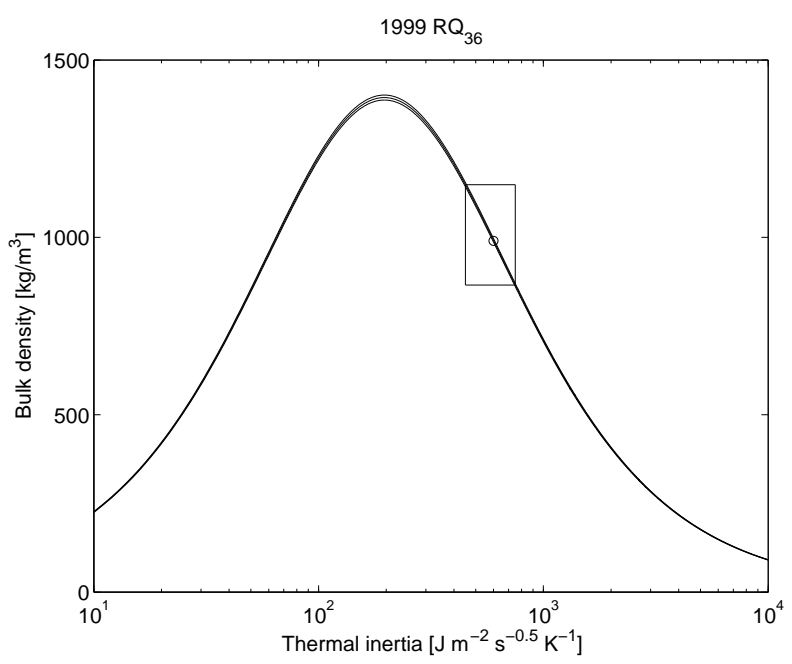

Figure 8: Density as a function of thermal inertia for asteroid $1999 \mathrm{RQ}_{36}$. The three lines corresponding to the nominal value and the 1- $\sigma$ levels are extremely narrow because of the high SNR. The circle corresponding to the nominal observed value of $\Gamma$ and thus inferred nominal value of $\rho$ (Cheslev et al., 2012) matches the plotted curves. The enclosed region corresponds to the 1- $\sigma$ interval for $\Gamma$ (Emery et al., 2012) and indicates how it maps onto the $1-\sigma$ limits of $\rho$.

latter two objects we assumed slope parameter $G=0.15$. Our findings are consistent with the taxonomic type. For instance, Golevka, Apollo, Toro, YORP, and Geographos are S/Q-type asteroids with an expected density between 2000 and $3000 \mathrm{~kg} / \mathrm{m}^{3}$, while Ra-Shalom is a C-type so we expect a bulk density from 500 and $1500 \mathrm{~kg} / \mathrm{m}^{3}$. Figure 9] suggests that Golevka has thermal inertia $150<\Gamma<500 \mathrm{~J} \mathrm{~m}^{-2} \mathrm{~s}^{-0.5} \mathrm{~K}^{-1}$ and Apollo has a rather large thermal inertia $400<\Gamma<1000$. According to Delbó et al. (2003) Ra-Shalom has an unusually high thermal inertia. In fact, by taking the right side of the plotted rectangle we obtain a density closer to $1000 \mathrm{~kg} / \mathrm{m}^{3}$, similar to the one $1999 \mathrm{RQ}_{36}$, which belongs to a similar taxonomic class. For Toro, Mueller (2012) reports a thermal inertia $200<\Gamma<1200 \mathrm{~J} \mathrm{~m}^{-2} \mathrm{~s}^{-0.5} \mathrm{~K}^{-1}$ but likely lower, which would result in a bulk density between 2000 and 4000 $\mathrm{kg} / \mathrm{m}^{3}$.

\subsection{Implications for impact predictions}

The question is how much the predictions of possible asteroid impacts on Earth can be affected by the Yarkovsky effect. There are already three well known examples, namely the asteroids Apophis, 1999 RQ $_{36}$, and 1950 DA 
for which Yarkovsky perturbations are relevant and need to be accounted for in the impact risk assessment.

For Apophis and $1999 \mathrm{RQ}_{36}$ this is due to the presence of a strongly scattering planetary close approach between now and the epochs of the possible impacts. These encounters transform a very well determined orbit into a poorly known one for which chaotic effects are dominant (Milani et al., 2009). Apophis will have a close approach in April 2029 with minimum distance of $\sim 38000 \mathrm{~km}$ from the geocenter. As a consequence the orbital uncertainty will increase by a factor $>40000$. $1999 \mathrm{RQ}_{36}$ will have a close approach to Earth in 2135 with nominal minimum distance about the same as the distance to the Moon, with an increase in uncertainty by a factor $\sim 500$. The minimum possible distance for this close approach is three times smaller and would result in an increase of the uncertainty by a factor $\sim 1500$ (Chesley et al., 2012). In both cases the Yarkovsky effect is large enough to shift the position at the scattering close approach by an amount much larger than the distance between the keyholes (Chodas, 1999) corresponding to impacts in later years (2036, 2037, 2068 for Apophis; 2175, 2180, 2196 for $\left.1999 \mathrm{RQ}_{36}\right)$. Thus, the occurrence of these later impacts is determined by the Yarkovsky perturbation in the years between now and the scattering encounter. For 1950 DA the influence of the Yarkovsky effect for the possible impact in 2880 is due to long time interval preceding the impact that allows the orbital displacement accumulate (Giorgini et al., 2002).

Currently, $1999 \mathrm{RQ}_{36}$ is the case with the best determined Yarkovsky effect (SNR 200), while Apophis and 1950 DA have only a marginal detection (SNR $<1$ and SNR $\sim 1.4$, respectively). Therefore, the impact monitoring for $1999 \mathrm{RQ}_{36}$ fully takes into account Yarkovsky (Chesley et al., 2012). On the contrary, the current estimate of impact probabilities for Apophis is based on a Montecarlo model of Yarkovsky based on a priori knowledge of the statistical properties of this effect (Chesley et al., 2009). 1950 DA could benefit from a similar approach. The knowledge of the Yarkovsky perturbation for Apophis is expected to increase dramatically due to observations possible during the January 2013 close approach.

We investigated the possibility that our identification of asteroids with measurable Yarkovsky produces new cases such as the two above, that is of impact monitoring affected by Yarkovsky. The answer to this question is negative, in that the intersection between the current list of NEA with possible impacts on Earth (337 according to NEODyS, 404 according to Sentry) and the list with detected Yarkovsky contains only $1999 \mathrm{RQ}_{36}$. 
However, this conclusion depends on the fact that our monitoring of possible future impacts is done for only about one century (currently 90 years for NEODyS, 100 for Sentry). $1999 \mathrm{RQ}_{36}$ was a special case, related to an intensified effort for the OSIRIS-REx mission target $1999 \mathrm{RQ}_{36}$ (http://osiris-rex.lpl.arizona.edu). If this time span were generally increased to 150-200 years, there could well be other cases similar to $1999 \mathrm{RQ}_{36}$.

For asteroid 1950 DA Busch et al. (2007) report a density around 3000 $\mathrm{km} / \mathrm{m}^{3}$ and two possible solutions for pole orientation and effective diameter:

1. $\gamma=24.47^{\circ}$ and $D=1.16 \mathrm{~km}$;

2. $\gamma=167.72^{\circ}$ and $D=1.30 \mathrm{~km}$.

By scaling from the $1999 \mathrm{RQ}_{36}$ case, we obtain $A_{2}=7.01 \mathrm{au} / \mathrm{d}^{2}$ for the direct solution and $A_{2}=-5.83 \mathrm{au} / \mathrm{d}^{2}$ for the retrograde solution. Even if we found a low SNR detection, our result strongly favors the retrograde solution, which is at $0.6 \sigma$, than the direct solution, which is more than $3 \sigma$ away. As a consequence, the 2880 impact would be ruled out (Giorgini et al., 2002).

\section{Conclusions}

In this paper we developed a 1-parameter formulation that models the transverse component of the Yarkovsky effect. Despite being simple, this formulation captures the essence the Yarkovsky effect and also allows us to solve for the introduced parameter as a part of the orbital fit.

The small magnitude of the Yarkovsky acceleration made it necessary to employ a high precision dynamical model including the effects of sixteen perturbing asteroids and planetary relativistic terms.

As the solution of the orbital fit depends on the observational data treatment, we applied the debiasing and weighting scheme by Chesley et al. (2010). Moreover, we resorted to some manual weighting in cases where an incorrect treatment non-CCD observations could have led to unreliable results.

After filtering out those objects with an unreasonably high orbital drift, we obtained 21 robust detections with a signal to noise ratio grater than 3. By connecting the sign of the orbital drift to the spin orientation, we found a 4:1 fraction of retrograde rotators. We discussed how this excess of retrograde rotators can be related to the delivery of NEAs to the inner Solar System.

For a few asteroids we were able to use the measured orbital drift along with the known physical characterization to constrain unknown physical quantities such as bulk density and thermal inertia. 
We also discussed the implication of the Yarkovsky effect and pointed out how this perturbation can be relevant either when there is a scattering close approach before the possible impact or when the impact is far enough in time.

\section{Acknowledgments}

DF was supported for this research in part by an appointment to the NASA Postdoctoral Program at the Jet Propulsion Laboratory, California Institute of Technology, administered by Oak Ridge Associated Universities through a contract with NASA, and in part by ESA/ESTEC-SpaceDyS Service Level Agreement SSA-NEO-ESA-SLA2-002_NEODYS.

$\mathrm{SC}$ conducted this research at the Jet Propulsion Laboratory, California Institute of Technology, under a contract with the National Aeronautics and Space Administration.

The work of DV was partially supported by the Czech Grant Agency (grant 205/08/0064) and Research Program MSM0021620860 of the Czech Ministry of Education.

\section{References}

Baer, J., Chesley, S. R., Matson, R. D., May 2011. Astrometric Masses of 26 Asteroids and Observations on Asteroid Porosity. The Astronomical Journal 141, 143.

Bottke, W. F., Morbidelli, A., Jedicke, R., Petit, J.-M., Levison, H. F., Michel, P., Metcalfe, T. S., Apr. 2002. Debiased Orbital and Absolute Magnitude Distribution of the Near-Earth Objects. Icarus 156, 399-433.

Bottke, Jr., W. F., Vokrouhlický, D., Rubincam, D. P., Nesvorný, D., May 2006. The Yarkovsky and Yorp Effects: Implications for Asteroid Dynamics. Annual Review of Earth and Planetary Sciences 34, 157-191.

Bowell, E., Hapke, B., Domingue, D., Lumme, K., Peltoniemi, J., Harris, A. W., 1989. Application of photometric models to asteroids. In: Binzel, R. P., Gehrels, T., Matthews, M. S. (Eds.), Asteroids II. pp. 524-556.

Brozović, M., Ostro, S. J., Benner, L. A. M., Giorgini, J. D., Jurgens, R. F., Rose, R., Nolan, M. C., Hine, A. A., Magri, C., Scheeres, D. J., Margot, 
J.-L., May 2009. Radar observations and a physical model of Asteroid 4660 Nereus, a prime space mission target. Icarus 201, 153-166.

Busch, M. W., Giorgini, J. D., Ostro, S. J., Benner, L. A. M., Jurgens, R. F., Rose, R., Hicks, M. D., Pravec, P., Kusnirak, P., Ireland, M. J., Scheeres, D. J., Broschart, S. B., Magri, C., Nolan, M. C., Hine, A. A., Margot, J.-L., Oct. 2007. Physical modeling of near-Earth Asteroid (29075) 1950 DA. Icarus 190, 608-621.

Čapek, D., Vokrouhlický, D., Dec. 2004. The YORP effect with finite thermal conductivity. Icarus 172, 526-536.

Carpino, M., Milani, A., Chesley, S. R., Dec. 2003. Error statistics of asteroid optical astrometric observations. Icarus 166, 248-270.

Chesley, S. R., Baer, J., Monet, D. G., Nov. 2010. Treatment of star catalog biases in asteroid astrometric observations. Icarus 210, 158-181.

Chesley, S. R., Chodas, P. W., Milani, A., Valsecchi, G. B., Yeomans, D. K., Oct. 2002. Quantifying the Risk Posed by Potential Earth Impacts. Icarus 159, 423-432.

Chesley, S. R., Milani, A., Tholen, D., Bernardi, F., Chodas, P., Micheli, M., Sep. 2009. An Updated Assessment Of The Impact Threat From 99942 Apophis. In: AAS/Division for Planetary Sciences Meeting Abstracts \#41. Vol. 41 of AAS/Division for Planetary Sciences Meeting Abstracts. p. \#43.06.

Chesley, S. R., Nolan, M. C., Farnocchia, D., Milani, A., Emery, J., Vokrouhlický, D., Lauretta, D. S., Taylor, P. A., Benner, L. A. M., Giorgini, J. D., Brozovic, M., Busch, M. W., Margot, J.-L., Howell, E. S., Naidu, S. P., Valsecchi, G. B., Bernardi, F., May 2012. The Trajectory Dynamics of Near-Earth Asteroid 101955 (1999 RQ36). LPI Contributions 1667, 6470.

Chesley, S. R., Ostro, S. J., Vokrouhlický, D., Čapek, D., Giorgini, J. D., Nolan, M. C., Margot, J.-L., Hine, A. A., Benner, L. A. M., Chamberlin, A. B., Dec. 2003. Direct Detection of the Yarkovsky Effect by Radar Ranging to Asteroid 6489 Golevka. Science 302, 1739-1742.

Chesley, S. R., Vokrouhlický, D., Ostro, S. J., Benner, L. A. M., Margot, J.L., Matson, R. L., Nolan, M. C., Shepard, M. K., 2008. Direct Estimation 
of Yarkovsky Accelerations on Near-Earth Asteroids. LPI Contributions 1405,8330 .

Chodas, P. W., 1999. Orbit uncertainties, keyholes, and collision probabilities. In: Bulletin of the American Astronomical Society. Vol. 31 of Bulletin of the American Astronomical Society. p. 1117.

Delbó, M., Dell'Oro, A., Harris, A. W., Mottola, S., Mueller, M., Sep. 2007. Thermal inertia of near-Earth asteroids and implications for the magnitude of the Yarkovsky effect. Icarus 190, 236-249.

Delbó, M., Harris, A. W., Binzel, R. P., Pravec, P., Davies, J. K., Nov. 2003. Keck observations of near-Earth asteroids in the thermal infrared. Icarus 166, 116-130.

Drummond, J. D., Wisniewski, W. Z., Feb. 1990. The rotational poles and shapes of 1580 Betulia and 3908 (1980PA) from one apparition. Icarus 83, 349-359.

Ďurech, J., Vokrouhlický, D., Baransky, A. R., Breiter, S., Burkhonov, O. A., Cooney, W., Fuller, V., Gaftonyuk, N. M., Gross, J., Inasaridze, R. Y., Kaasalainen, M., Krugly, Y. N., Kvaratshelia, O. I., Litvinenko, E. A., Macomber, B., Marchis, F., Molotov, I. E., Oey, J., Polishook, D., Pollock, J., Pravec, P., Sárneczky, K., Shevchenko, V. G., Slyusarev, I., Stephens, R., Szabó, G., Terrell, D., Vachier, F., Vanderplate, Z., Viikinkoski, M., Warner, B. D., Nov. 2012. Analysis of the rotation period of asteroids (1865) Cerberus, (2100) Ra-Shalom, and (3103) Eger - search for the YORP effect. Astronomy \& Astrophysics 547, A10.

Ďurech, J., Vokrouhlický, D., Kaasalainen, M., Higgins, D., Krugly, Y. N., Gaftonyuk, N. M., Shevchenko, V. G., Chiorny, V. G., Hamanowa, H., Hamanowa, H., Reddy, V., Dyvig, R. R., Oct. 2008a. Detection of the YORP effect in asteroid (1620) Geographos. Astronomy \& Astrophysics 489, L25-L28.

Ďurech, J., Vokrouhlický, D., Kaasalainen, M., Weissman, P., Lowry, S. C., Beshore, E., Higgins, D., Krugly, Y. N., Shevchenko, V. G., Gaftonyuk, N. M., Choi, Y.-J., Kowalski, R. A., Larson, S., Warner, B. D., Marshalkina, A. L., Ibrahimov, M. A., Molotov, I. E., Michałowski, T., Kitazato, K., Sep. 2008b. New photometric observations of asteroids (1862) Apollo and 
(25143) Itokawa - an analysis of YORP effect. Astronomy \& Astrophysics $488,345-350$.

Emery, J. P., Kelley, M. S., Fernandez, Y. R., Hergenrother, C. W., Crane, K. T., Ziffer, J., Campins, H., Lauretta, D. S., Drake, M. J., Oct. 2012. Thermal and Physical Characterization of the OSIRIS-REx Target Asteroid (101955) 1999 RQ36. In: AAS/Division for Planetary Sciences Meeting Abstracts. Vol. 44 of AAS/Division for Planetary Sciences Meeting Abstracts. p. \#102.05.

Gehrels, T., Roemer, E., Taylor, R. C., Zellner, B. H., Mar. 1970. Minor planets and related objects. IV. Asteroid (1566) Icarus. The Astronomical Journal 75, 186-195.

Giorgini, J. D., Benner, L. A. M., Ostro, S. J., Nolan, M. C., Busch, M. W., Jan. 2008. Predicting the Earth encounters of (99942) Apophis. Icarus 193, $1-19$.

Giorgini, J. D., Ostro, S. J., Benner, L. A. M., Chodas, P. W., Chesley, S. R., Hudson, R. S., Nolan, M. C., Klemola, A. R., Standish, E. M., Jurgens, R. F., Rose, R., Chamberlin, A. B., Yeomans, D. K., Margot, J.-L., Apr. 2002. Asteroid 1950 DA's Encounter with Earth in 2880: Physical Limits of Collision Probability Prediction. Science 296, 132-136.

Hudson, R. S., Ostro, S. J., Jurgens, R. F., Rosema, K. D., Giorgini, J. D., Winkler, R., Rose, R., Choate, D., Cormier, R. A., Franck, C. R., Frye, R., Howard, D., Kelley, D., Littlefair, R., Slade, M. A., Benner, L. A. M., Thomas, M. L., Mitchell, D. L., Chodas, P. W., Yeomans, D. K., Scheeres, D. J., Palmer, P., Zaitsev, A., Koyama, Y., Nakamura, A., Harris, A. W., Meshkov, M. N., Nov. 2000. Radar Observations and Physical Model of Asteroid 6489 Golevka. Icarus 148, 37-51.

Konopliv, A. S., Asmar, S. W., Folkner, W. M., Karatekin, Ö., Nunes, D. C., Smrekar, S. E., Yoder, C. F., Zuber, M. T., Jan. 2011. Mars high resolution gravity fields from MRO, Mars seasonal gravity, and other dynamical parameters. Icarus 211, 401-428.

La Spina, A., Paolicchi, P., Kryszczyńska, A., Pravec, P., Mar. 2004. Retrograde spins of near-Earth asteroids from the Yarkovsky effect. Nature 428, 400-401. 
Marsden, B. G., Sekanina, Z., Yeomans, D. K., Mar. 1973. Comets and nongravitational forces. V. The Astronomical Journal 78, 211.

Micheli, M., Tholen, D. J., Elliott, G. T., May 2012. Detection of radiation pressure acting on 2009 BD. New Astronomy 17, 446-452.

Mignard, F., 2003. Observations of Small Solar System Bodies with GAIA. In: IAU Joint Discussion. Vol. 19 of IAU Joint Discussion.

Milani, A., Chesley, S. R., Sansaturio, M. E., Bernardi, F., Valsecchi, G. B., Arratia, O., Oct. 2009. Long term impact risk for (101955) 1999 RQ. Icarus 203, 460-471.

Moyer, T. D., 2003. Formulation for Observed and Computed Values of Deep Space Network Data Types for Navigation. Wiley-Interscience, Hoboken, NJ.

Mueller, M., Aug. 2012. Surface Properties of Asteroids from Mid-Infrared Observations and Thermophysical Modeling. ArXiv e-prints.

Nugent, C. R., Mainzer, A., Masiero, J., Grav, T., Bauer, J., Sep. 2012a. The Yarkovsky Drift's Influence on NEAs: Trends and Predictions with NEOWISE Measurements. The Astronomical Journal 144, 75.

Nugent, C. R., Margot, J. L., Chesley, S. R., Vokrouhlický, D., Aug. 2012b. Detection of Semimajor Axis Drifts in 54 Near-Earth Asteroids: New Measurements of the Yarkovsky Effect. The Astronomical Journal 144, 60.

Pravec, P., Harris, A. W., Sep. 2007. Binary asteroid population. 1. Angular momentum content. Icarus 190, 250-259.

Rozitis, B., Green, S. F., Jun. 2012. The influence of rough surface thermalinfrared beaming on the Yarkovsky and YORP effects. Monthly Notices of the Royal Astronomical Society 423, 367-388.

Standish, E. M., 2000. Recommendation of DE405 for 2001 Mars Surveyor and for Cassini. Tech. Rep. IOM 312.F-00-107b, Jet Propulsion Laboratory.

Standish, E. M., Hellings, R. W., Aug. 1989. A determination of the masses of Ceres, Pallas, and Vesta from their perturbations upon the orbit of Mars. Icarus 80, 326-333. 
Taylor, P. A., Margot, J.-L., Vokrouhlický, D., Scheeres, D. J., Pravec, P., Lowry, S. C., Fitzsimmons, A., Nolan, M. C., Ostro, S. J., Benner, L. A. M., Giorgini, J. D., Magri, C., Apr. 2007. Spin Rate of Asteroid (54509) 2000 PH5 Increasing Due to the YORP Effect. Science 316, 274-.

Vokrouhlický, D., Jul. 1998. Diurnal Yarkovsky effect as a source of mobility of meter-sized asteroidal fragments. I. Linear theory. Astronomy \& Astrophysics 335, 1093-1100.

Vokrouhlický, D., Apr. 1999. A complete linear model for the Yarkovsky thermal force on spherical asteroid fragments. Astronomy \& Astrophysics $344,362-366$.

Vokrouhlický, D., Chesley, S. R., Matson, R. D., Jun. 2008. Orbital Identification for Asteroid 152563 (1992 Bf) Through the Yarkovsky Effect. The Astronomical Journal 135, 2336-2340.

Vokrouhlický, D., Milani, A., Chesley, S. R., Nov. 2000. Yarkovsky Effect on Small Near-Earth Asteroids: Mathematical Formulation and Examples. Icarus 148, 118-138.

Will, C. M., Mar. 1993. Theory and Experiment in Gravitational Physics. Cambridge University Press.

Yeomans, D. K., Antreasian, P. G., Barriot, J.-P., Chesley, S. R., Dunham, D. W., Farquhar, R. W., Giorgini, J. D., Helfrich, C. E., Konopliv, A. S., McAdams, J. V., Miller, J. K., Owen, W. M., Scheeres, D. J., Thomas, P. C., Veverka, J., Williams, B. G., Sep. 2000. Radio Science Results During the NEAR-Shoemaker Spacecraft Rendezvous with Eros. Science 289, 2085-2088. 
Table 2: Semimajor axis, eccentricity, absolute magnitude, physical and Yarkovsky parameters, and observational information for selected NEAs. The physical quantities for $1999 \mathrm{RQ}_{36}$ are from Cheslev et al. (2012). Golevka's obliquity $\gamma$ is from Hudson et al. (2000), Apollo's from Ďurech et al. (2008b), Nyx's from Drummond and Wisniewski (1990), Ra-Shalom's from Durech et al. (2012), YORP's from Tavlor et al. (2007), Geographos' from Durech et al. (2008a), and Toro's from Durech (personal communication). Diameters $D$ and rotation periods $P$ are from the European Asteroid Research Node (EARN) database (http://earn.dlr.de/nea/). Bond albedo $A$ was computed from geometric albedo $p_{V}$ and slope parameter $G$ (both from the EARN database when available) by $A=(0.290+0.684 G) p_{V}$ (Bowell et al., 1989).

\begin{tabular}{|c|c|c|c|c|c|c|c|c|c|c|c|c|c|c|c|}
\hline Asteroid & $\begin{array}{c}a \\
{[\mathrm{au}]}\end{array}$ & $e$ & $\bar{H}$ & $\begin{array}{c}D \\
\mathrm{~km}]\end{array}$ & $\begin{array}{c}P \\
{[\mathrm{hr}]}\end{array}$ & $\mathrm{A}$ & $\gamma$ & $\begin{array}{c}A_{2} \\
{\left[10^{-15} \mathrm{au} / \mathrm{d}^{2}\right]}\end{array}$ & $\begin{array}{l}\text { SNR } \\
\end{array}$ & $\overline{\mathcal{S}}$ & $\begin{array}{c}d a / d t \\
{\left[10^{-4} \mathrm{au} / \mathrm{Myr}\right]}\end{array}$ & $\begin{array}{c}d a / d t \\
\text { Nugent et al. }\end{array}$ & $\begin{array}{c}\text { Diff. } \\
\sigma\end{array}$ & $\begin{array}{l}\text { Observed } \\
\text { arc }\end{array}$ & $\begin{array}{c}\text { Radar } \\
\text { apparitions }\end{array}$ \\
\hline $\begin{array}{l}(101955) \\
1999\end{array} \mathrm{RQ}_{36}$ & 1.13 & 0.20 & 20.6 & 0.49 & 4.29 & 0.01 & $175^{\circ}$ & $-45.49 \pm 0.23$ & 197.7 & 1.0 & $-18.99 \pm 0.10$ & $-18.9 \pm 0.2$ & 0.4 & $1999-2012$ & $\begin{array}{c}1999,2005 \\
2011\end{array}$ \\
\hline 52563) 19 & 91 & 27 & .6 & 0.51 & NA & 0.02 & & $24.01 \pm$ & 19.8 & 0.5 & $-11.55 \pm 0$ & $-12.84 \pm 1$ & 1.1 & $1953-$ & NA \\
\hline 189) Colo & & 60 & 9.1 & 0.27 & 6.03 & 0.23 & & $1588+$ & 10.4 & 0.2 & $-6.62 \pm 0$ & $.74 \pm 0.7$ & 0.9 & & $\begin{array}{c}1991,1995 \\
2003\end{array}$ \\
\hline 2009 BD & 1.01 & .04 & 28.2 & NA & NA & NA & NA & $-1164.01 \pm 138.76$ & 8.3 & 0.4 & $-493.39 \pm 58.81$ & NA & NA & 2009-2011 & NA \\
\hline Apollo & 1.47 & .56 & 16.0 & 1.40 & 3.06 & 0.12 & $62^{\circ}$ & $4 \pm 0$ & 6.4 & 0.2 & $-1.70 \pm 0$ & $-2.3 \pm 0.2$ & 1.8 & 012 & 1980 \\
\hline 062) Aten & 97 & 18 & 6.9 & 1.30 & 0.77 & NA & & & 6.3 & 0.9 & -6.2 & $-7.5 \pm$ & 0.4 & & 12 \\
\hline (3908) Nyx & 93 & 46 & 7.3 & 1.00 & 4.43 & NA & & \pm & 5.6 & 1.3 & 11.6 & $12.9 \pm 2.7$ & 0.4 & 012 & 04 \\
\hline (2100) Ra-Shalom & 0.83 & 0.44 & 16.1 & 2.24 & 19.80 & 0.05 & $162^{\circ}$ & $-10.97 \pm$ & 4.9 & 1.1 & & $-5.4 \pm 1.5$ & 0.5 & $1975-2012$ & $\begin{array}{l}1981,1984, \\
2000,2003\end{array}$ \\
\hline 10302) 19 & 1.27 & 14 & 9.4 & 0.25 & 19 & NA & NA & +1 & 4.6 & 0 & \pm 6.28 & $35.3 \pm 7.1$ & 0.0 & 012 & NA \\
\hline & & 67 & 1.4 & NA & 5.49 & NA & & & 4.5 & .4 & & NA & NA & & \\
\hline Hat & & 45 & 9.9 & 0.30 & NA & 0.24 & & & 4.4 & 0.3 & & $-14.5 \pm 3.5$ & 0.0 & & \\
\hline 6037) 198 & 1.27 & 50 & 18.7 & 0.40 & 2.76 & NA & $\mathrm{N}$ & \pm & 4.0 & 0.6 & -16 & $-14.2 \pm 4.3$ & 0.4 & 011 & 8 \\
\hline 37655) Il & & 75 & 7.8 & NA & 2.66 & NA & & 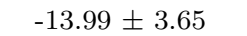 & .8 & 0.6 & -11 & NA & NA & & \\
\hline (85953) $1999 \mathrm{FK}_{21}$ & 0.74 & 0.70 & 18.0 & 0.59 & NA & NA & NA & $-10.62 \pm 2$ & 3.7 & 0.3 & $-10.38 \pm 2.28$ & $-10.44 \pm 1.5$ & 0.0 & 012 & NA \\
\hline (1685) Toro & 1.37 & 0.44 & 14.3 & 3.00 & 10.20 & 0.05 & $144^{\circ}$ & $-2.83 \pm 0.77$ & 3.7 & 0.4 & $-1.27 \pm 0.34$ & $-1.4 \pm 0.7$ & 0.2 & $1948-2012$ & $\begin{array}{c}1980,1988 \\
2012\end{array}$ \\
\hline & & & & & & & & & & & & & NA & & \\
\hline & & & 6 & 0. & 0.20 & & & & & & & $35.63 \pm$ & 0.1 & & 2001, 2004 \\
\hline (283457) 20( & 2.23 & 46 & 3.9 & NA & NA & NA & $\mathrm{N}$ & $-44.63 \pm 14$ & 3.1 & 1.1 & $-16.02 \pm 5.14$ & NA & NA & 51-2011 & NA \\
\hline (1620) Geogra & 1.25 & 0.34 & 5.2 & 2.47 & 5.22 & NA & $153^{\circ}$ & $-4.25 \pm 1$ & 3.0 & 0.5 & $-1.82 \pm 0.60$ & $-2.5 \pm 0.6$ & 0.8 & 1951-2012 & 1983, 1994 \\
\hline (65679) 1989 UQ & 0.91 & 0.26 & 19.3 & 0.73 & 7.73 & NA & NA & $-36.66 \pm 12.23$ & 3.0 & 1.2 & $-17.51 \pm 5.84$ & NA & NA & & NA \\
\hline (162004) $1991 \mathrm{VE}$ & 0.89 & 0.66 & 18.0 & NA & NA & NA & NA & $18.30 \pm 6.13$ & 3.0 & 0.7 & $14.75 \pm 4.94$ & NA & NA & 1954-2012 & NA \\
\hline
\end{tabular}


Table 3: Same as Table 2 for less reliable detections. Icarus' obliquity is from Gehrels et al. (1970), Eros' from Yeomans et al. (2000), Nereus' from Brozović et al. (2009), and Betulia's from Drummond and Wisniewski (1990). All the other physical quantities are from the EARN database.

\begin{tabular}{|c|c|c|c|c|c|c|c|c|c|c|c|c|c|c|c|}
\hline Asteroid & $\begin{array}{c}a \\
\mathrm{au}]\end{array}$ & $\bar{e}$ & $\bar{H}$ & $\begin{array}{c}D \\
{[\mathrm{~km}]}\end{array}$ & $\begin{array}{c}P \\
{[\mathrm{hr}]}\end{array}$ & $\overline{\mathrm{A}}$ & $\gamma$ & $\begin{array}{c}A_{2} \\
{\left[10^{-15} \mathrm{au} / \mathrm{d}^{2}\right]}\end{array}$ & SNR & $\overline{\mathcal{S}}$ & $\begin{array}{c}d a / d t \\
{\left[10^{-4} \mathrm{au} / \mathrm{Myr}\right]}\end{array}$ & $\begin{array}{c}d a / d t \\
\text { Nugent et al. }\end{array}$ & $\begin{array}{l}\text { Diff. } \\
\sigma\end{array}$ & $\begin{array}{l}\text { Observed } \\
\text { arc }\end{array}$ & $\begin{array}{c}\text { Radar } \\
\text { apparitions }\end{array}$ \\
\hline $1999 \mathrm{FA}$ & 1.07 & 0.13 & 20.6 & 0.30 & 10.09 & $\mathrm{NA}$ & $\mathrm{NA}$ & $-98.69 \pm 6.15$ & 3.2 & 1.3 & $-41.07 \pm 13.02$ & NA & $\overline{\mathrm{NA}}$ & $1978-2008$ & NA \\
\hline (433) Eros & 1.46 & 0.22 & 10.8 & 23.30 & 5.27 & 0.10 & $89^{\circ}$ & $-0.72 \pm 0.23$ & 3.1 & 0.7 & $-0.26 \pm 0.09$ & $-0.3 \pm 0.2$ & 0.2 & $1893-2012$ & 1975,1988 \\
\hline (4660) Nereus & 1.49 & 0.36 & 18.1 & 0.34 & 15.16 & NA & $11^{\circ}$ & $28.58 \pm 11.72$ & 2.4 & 0.4 & $11.43 \pm 4.69$ & $10.9 \pm 4.8$ & 0.1 & $1981-2012$ & 2001,2002 \\
\hline $2007 \mathrm{~PB}_{8}$ & 0.88 & 0.45 & 21.2 & NA & NA & NA & NA & $-156.01 \pm 66.45$ & 2.3 & 1.4 & $-88.05 \pm 37.50$ & NA & NA & $2002-2012$ & NA \\
\hline $2004 \mathrm{BG}_{41}$ & 2.52 & 0.61 & 24.4 & NA & NA & NA & NA & $-598.99 \pm 255.87$ & 2.3 & 1.2 & $-256.03 \pm 109.37$ & NA & NA & $2004-2011$ & NA \\
\hline (4179) Toutatis & 2.53 & 0.63 & 15.1 & 2.80 & 176.00 & 0.05 & NA & $-3.32 \pm 1.43$ & 2.3 & 0.4 & $-1.49 \pm 0.63$ & $-5.0 \pm 0.6$ & 4.1 & $1976-2012$ & $\begin{array}{c}1992,1996, \\
2000,2004, \\
2008\end{array}$ \\
\hline (138911) $2001 \mathrm{AE}_{2}$ & 1.35 & 0.08 & 19.0 & 0.35 & NA & NA & NA & $-62.23 \pm 28.84$ & 2.2 & 1.0 & $-22.90 \pm 10.61$ & $-22.9 \pm 11.2$ & 0.0 & $1984-2012$ & NA \\
\hline (326290) $1998 \mathrm{HE}_{3}$ & 0.88 & 0.44 & 21.7 & 0.10 & NA & NA & NA & $-70.57 \pm 33.01$ & 2.1 & 0.3 & $-39.68 \pm 18.56$ & NA & NA & $1993-2012$ & 2012 \\
\hline (3361) Orpheus & 1.21 & 0.32 & 18.9 & 0.50 & 3.51 & NA & NA & $12.07 \pm 5.67$ & 2.1 & 0.3 & $5.20 \pm 2.44$ & $5.7 \pm 2.5$ & 0.1 & $1982-2010$ & NA \\
\hline (154590) $2003 \mathrm{MA}_{3}$ & 1.11 & 0.40 & 21.7 & NA & NA & NA & NA & $-95.60 \pm 45.11$ & 2.1 & 0.7 & $-46.06 \pm 21.74$ & NA & NA & $1998-2012$ & NA \\
\hline $1994 \mathrm{XL}_{1}$ & 0.67 & 0.53 & 20.8 & NA & NA & NA & NA & $-31.22 \pm 15.32$ & 2.0 & 0.3 & $-22.38 \pm 10.98$ & NA & NA & 1994-2011 & NA \\
\hline (1580) Betulia & 2.19 & 0.49 & 14.3 & 4.57 & 6.13 & 0.03 & $117^{\circ}$ & $-4.65 \pm 2.31$ & 2.0 & 0.9 & $-1.75 \pm 0.87$ & $-1.3 \pm 0.9$ & 0.4 & $1950-2012$ & $\begin{array}{c}1976,1989, \\
2002\end{array}$ \\
\hline (29075) $1950 \mathrm{DA}$ & 1.70 & 0.51 & 17.0 & 1.4 & 2.12 & 0.08 & NA & $-5.00 \pm 3.47$ & 1.4 & 0.3 & $-2.20 \pm 1.52$ & NA & NA & $1950-2012$ & 2001 \\
\hline (250680) $2005 \mathrm{QC}_{5}$ & 0.89 & 0.36 & 19.7 & NA & NA & NA & NA & $26.44 \pm 22.01$ & 1.2 & 0.5 & $13.70 \pm 11.40$ & NA & NA & $1978-2011$ & NA \\
\hline (105140) $2000 \mathrm{NL}_{10}$ & 0.91 & 0.82 & 15.5 & 1.72 & 6.93 & NA & NA & $-11.78 \pm 10.49$ & 1.1 & 0.9 & $-15.73 \pm 14.02$ & NA & NA & $1951-2012$ & NA \\
\hline (1566) Icarus & 1.08 & 0.83 & 16.7 & 1.30 & 2.27 & 0.04 & $76^{\circ}$ & $-0.66 \pm 1.39$ & 0.5 & 0.1 & $-0.86 \pm 1.80$ & $-3.2 \pm 2.0$ & 0.9 & $1949-2012$ & 1968,1996 \\
\hline
\end{tabular}



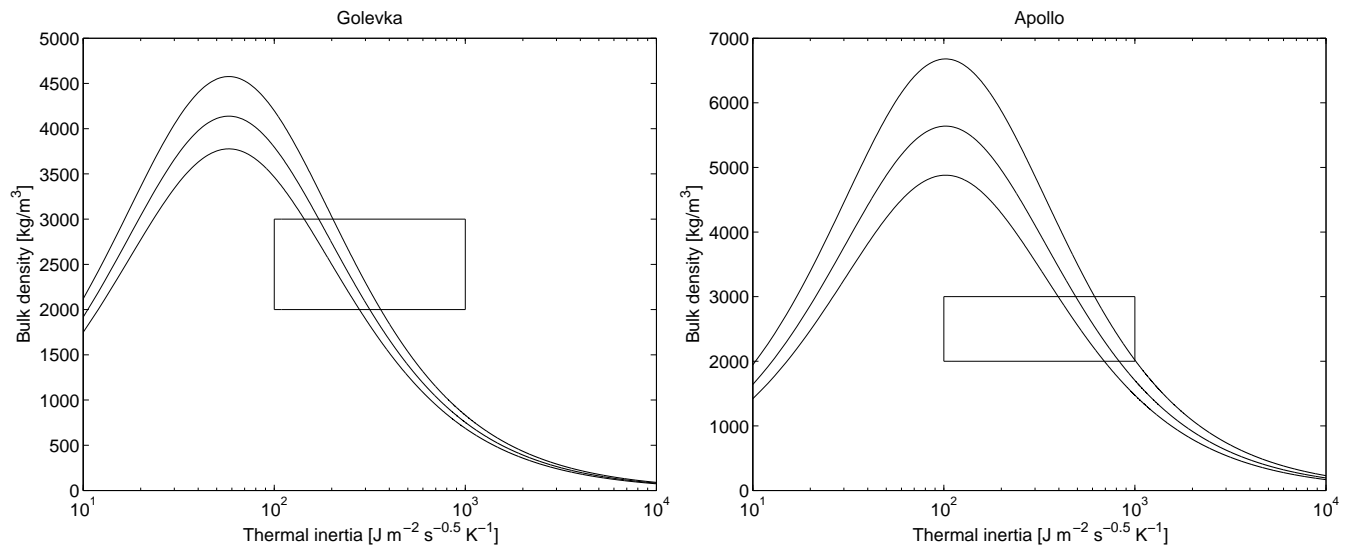

Ra-Shalom
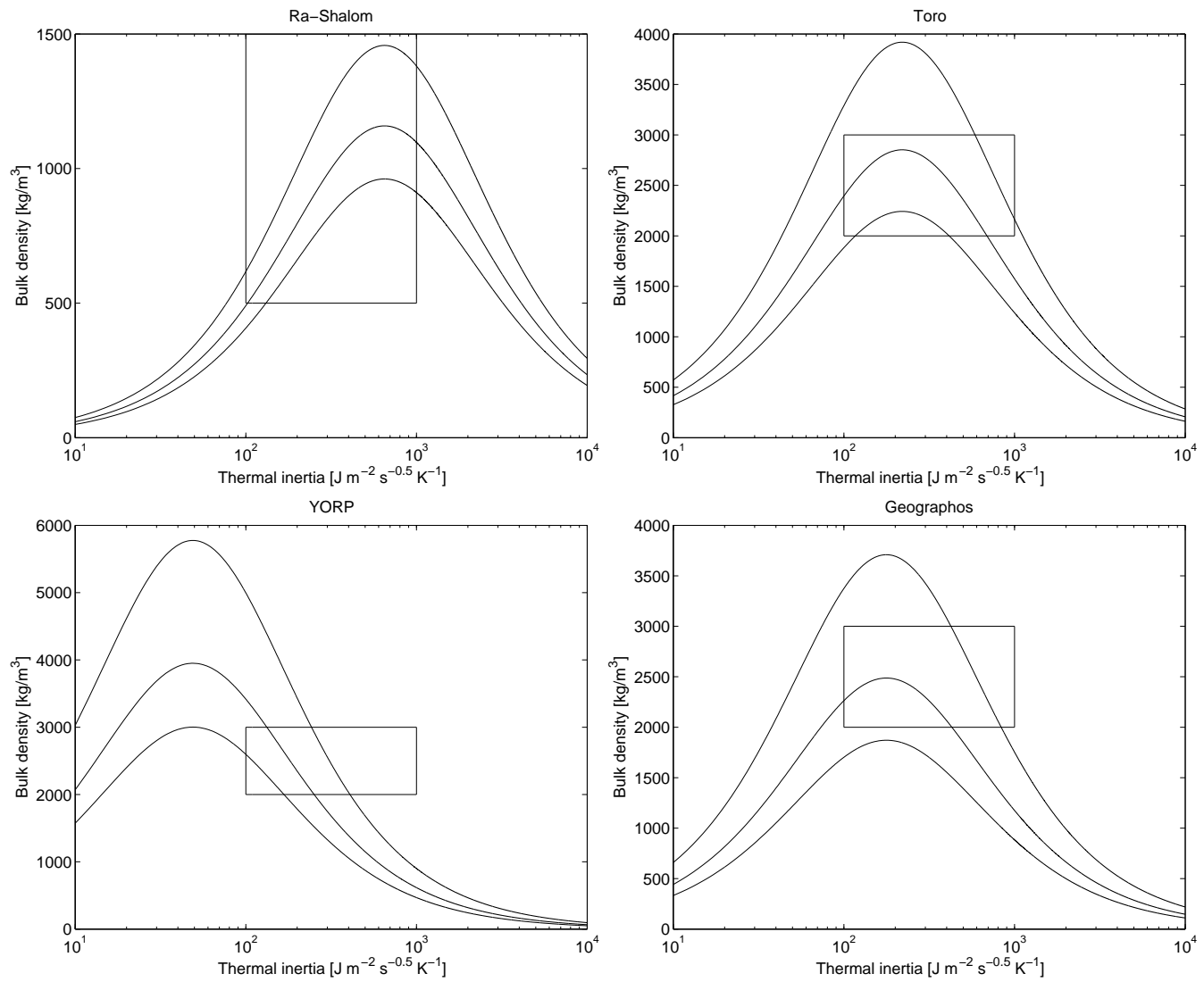

Figure 9: Density as a function of thermal inertia for asteroids Golevka, Apollo, RaShalom, Toro, YORP, and Geographos. The rectangles correspond to reasonable values of $\rho$ according to the taxonomic type and to a reasonable range of $\Gamma$ (Delbó et al., 2007). 